Polymer Journal, Vol. 8, No. 5, pp 391-400 (1976)

\title{
Fracture Processes in Ductile Polymer. II. Morphological Analysis of the Localized Plastic Deformation of Polycarbonate Film
}

\author{
Masaru Ishikawa, Ikuo Narisawa, and Hiroyuki OgaWa \\ Faculty of Engineering, Yamagata University, \\ 4-3-16, Yonezawa 992, Japan.
}

(Received October 6, 1975)

\begin{abstract}
The plastic deformation behavior of an artificially induced crack tip in polycarbonate films has been studied morphologically. It is shown that the plasticzone ahead of the crack tip consists of crazes, shear bands and their intersections.

While the craze initiation is restricted to the immediate vicinity of the crack tip, the shear bands extend to a region some distance from the initial crack tip and their intersections closely relate to the local reduction in thickness of the specimen.

The structure of the shear bands and their intersections which are associated with the biaxial stresses at the plastic-zone tip causes biaxial orientation of molecular chains.

The plastic strain in the intersection of the shear bands is discussed on the basis of the concept of the strain ellipsoid, and it is suggested that deformation caused by the intersection of the shear bands results from reorientation of the molecular chains initially oriented by the first shear rather than the duplication of simple shear deformations.

KEY WORDS Plastic-zone / Shear Band / Craze / Polycarbonate / Simple Shear / Strain Ellipsoid / Biaxial Orientation /
\end{abstract}

It is now well known that linear fracture mechanics can be usefully applied to the fracture behaviors of brittle polymers. For example, when examining the fracture of cracked specimens of poly(methyl methacrylate), provided the dimensions of the plastic-zone are small enough in comparison with the crack size, it is permissible to calculate stress distribution and to derive the stress intensity factor by assuming linear elasticity of the material. On the other hand, in many practical cases, the majority of polymers are ductile and the sizes of the plastic-zone cannot be neglected. In these polymers, the dimensions of the plastic-zone usually grow to several times as large as the crack length. Therefore, it should be mentioned that analysis based on linear fracture mechanics can be in significant disagreement with experiments.

According to a fracture criterion derived from nonlinear fracture mechanics, the fracture initiation depends strongly on the plastic-zone size ahead of the crack tip. Therefore, it is important to have an accurate estimate of the plastic-zone size. The Dugdale model, one of the typical nonlinear models, assumes that the material ahead of the crack tip yields at a constant stress equal to the uniaxial tensile yield stress. This assumes that the state of stress around the crack tip is a plane stress condition and the yielding of the material obeys the Tresca yield criterion. In the first paper $^{1}$ of this series the applicability of the Dugdal model to polycarbonate and nylon was studied and it was concluded that they could be compared well with the modified Dugdale model where the cohesive stresses varied linearly along the crack surface. Sternstein and Ongchin ${ }^{2,3}$ have propose that the yielding of glassy polymers obeys a pressure-dependent Mises yield criterion (modified von Mises criterion). This difference in the yield criterion of glassy polymers should be examined because, as mentioned before, the shapes of the plastic-zone depend strongly on the yield criterion which is applied to the material under consideration.

On the other hand, Vincent ${ }^{4}$ pointed out that the development of the plastic-zone ahead of the 
crack tip depended on the load-extension curve and he concluded that the plastic-zone shapes around the crack were severely influenced by the rate of hardening.

The purpose of this paper is to explain morphologically the structure of plastic-zone in the vicinity of the crack and to discuss the initiation of plastic deformation in connection with molecular orientation.

\section{EXPERIMENTAL}

\section{Material}

The material used was a commercial grade of polycarbonate, Panlite, (Teijin Co. Ltd). The specimens were cut from extruded sheets, $10 \mathrm{~mm}$ wide and $270 \mathrm{~mm}$ long. In the center of one edge of the specimen, a crack of $0.2-0.3 \mathrm{~mm}$ length was introduced by driving a sharp knife. The thickness of the specimens was as follows:

$0.1 \mathrm{~mm}$ : For observation of the plasticzone shape around the crack.

$0.3-0.5 \mathrm{~mm}$ : For observation of the crosssections of the plastic-zone and analysis of photoelasticity around the plastic-zone.

$0.03 \mathrm{~mm}$ : For measurement of the birefringence of the plastic-zone.

The strips $5 \mathrm{~mm}$ wide, $270 \mathrm{~mm}$ long and $30 \mu$ thick were used to determine the load-extension curve for the uncracked specimens. All specimens were annealed for 3 hours at the glassy transition temperature $\left(152^{\circ} \mathrm{C}\right)$ to eliminate the residual strain and cooled slowly to room temperature at a rate of $10^{\circ} \mathrm{C} / \mathrm{hr}$.

\section{Apparatus and Test Method}

The specimens were loaded by a tensile testing machine with which it was convenient to use a polarizing microscope. The tensile tests were carried out at a constant strain rate of $0.1 \% / \mathrm{min}$ for the cracked specimens and $0.4 \% / \mathrm{min}$ for the uncracked specimens, respectively. All measurements were made in air at $20^{\circ} \mathrm{C}$ and $65 \% \mathrm{RH}$.

Microscopic observations were made on the specimens under tension. Successive series of thin cross-section were cut from the deformed specimens to examine in a polarizing microscope. The cutting directions were perpendicular and parallel to the progressive direction of the plasticzone as shown in Figure 1. The photoelastic

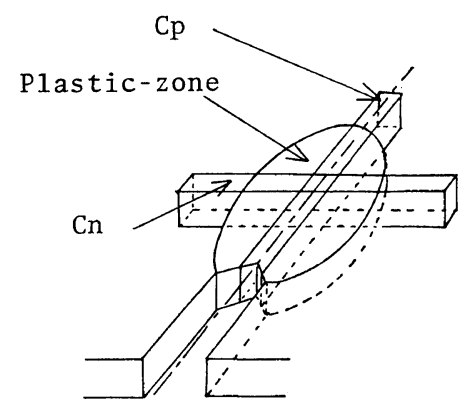

Figure 1. Cutting direction of the thin cross-sections: $\mathrm{Cn}$, perpendicular to the progressive direction of the plastic-zone, $\mathrm{Cp}$, parallel to the progressive direction of the plastic-zone.

observations were made by the aid of a polarizing microscope where a pair of $\lambda / 4$ plates was inserted.

\section{RESULTS}

\section{Shape of the Plastic-Zone}

The most accurate method of determining the plastic-zone shape around the crack tip is to observe the residual birefringence. In the case of a large plastic-zone, however, the shape varies on removing the stress so that the accurate shape of the plastic-zone is difficult to determine. Therefore, the shape of plastic-zone should be observed directly under loading. Brinson ${ }^{5}$ and Vincent, ${ }^{4}$ independently, have identified the shape of plastic-zone with the isochromatic fringe contours, but this method is inadequate for the objectives of this study for reasons explained briefly in a previous paper. ${ }^{1}$ For example,

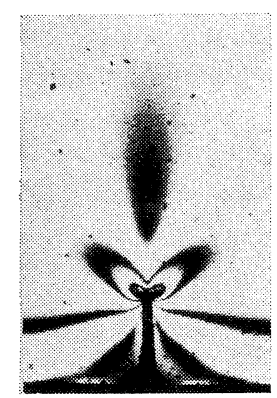

(A)

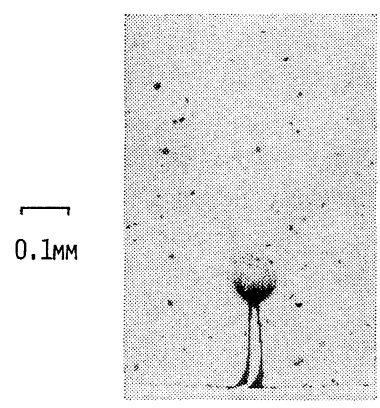

(B)
Figure 2. Comparison of the shape of the plasticzone (B) with isochromatic fringes (A) around the crack tip at a low stress level. 


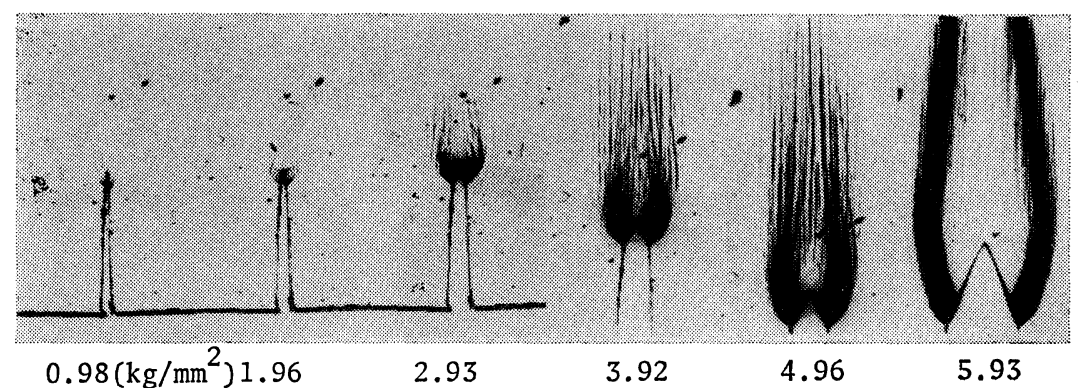

Figure 3. Successive microphotographs of the shape of the plastic-zone on increasing the stress level.

in the case of a small plastic-zone, although the distribution of isochromatic fringes around the crack tip is similar to that observed on an entirely elastic material, the plastic-zone near the initial crack tip has a shape clearly different from the isochromatic fringe contours (Figures 2(A) and $2(\mathrm{~B})$ ).

Figure 3 shows successive microphotographs of the plastic-zone at various stress levels. At smaller stress levels $\left(1-2 \mathrm{~kg} / \mathrm{mm}^{2}\right)$ propagation of the crack does not occur, but plastic deformation initiates with the development of the dark deformation-zone at the crack tip. As the stress is increased, this zone propagates perpendicular to the tensile axis, but the width does not develop. At a stress of about $2.0 \mathrm{~kg} / \mathrm{mm}^{2}$ fine lines appear around the dark deformationzone. As the stress is increased, these lines propagate nearly perpendicular to the tensile axis and in this stage of deformation, the plasticzone is composed of both the fine lines and the dark deformation-zone. At a stress of about $3.0 \mathrm{~kg} / \mathrm{mm}^{2}$, growth of the dark deformationzone practically stops and only the fine lines continue to grow. In the range of 3.5-4.6 $\mathrm{kg} / \mathrm{mm}^{2}$ growth of the dark deformation-zone stops completely and the fine lines become more intense. At this stage, the crack begins to grow as may be apparent from the increase in the crack-opening displacement near the initial crack tip. When the stress is increased to $4.7 \mathrm{~kg} / \mathrm{mm}^{2}$ the growing crack passes throughout the dark deformation-zone and the fine lines at the crack tip become invisible and a homogenous zone appears around the crack tip. When the stress is increased to $5.27 \mathrm{~kg} / \mathrm{mm}^{2}$, the homogenous zone extends and the fine lines concentrate to the boundary of the plastic-zone. Further stressing leads to catastrophic failure of the specimen.

\section{Cross-Sections of the Plastic-Zone}

Though the deformation processes around the crack tip are affected by both the initial crack length and thickness of the specimen, the fundamental process can be divided into two steps. As the first step, the dark deformation-zone and the fine lines develop but no propagation of the initial crack occurs. As the second step, the homogenous deformation region is formed and the fine lines concentrate to the boundary of the plastic-zone.

Figure 4 shows the variation of the appearance of the cross-section with distance from the crack tip in the first step. Both the perpendicular and inclined deformation bands to the tensile axis can be observed. A comparison of the crosssection with the overall view of the plastic-zone indicates that the dark deformation zone corresponds to the perpendicular deformation-band and that each fine line is the edge of the inclined deformation-band. The zone of the perpendicular deformation-band diminishes in size and the inclined deformation-bands extend into the interior of the specimen on leaving the crack tip. The perpendicular deformation-band becomes invisible at a distance of $0.18 \mathrm{~mm}$ from the crack tip and only the inclined deformation-bands can be observed. A slight decrease in thickness of the specimen can be observed at distances of $0.03-0.08 \mathrm{~mm}$.

Judging from the extinction direction, the perpendicular deformation-band has an oriented structure which is parallel to the tensile axis and each inclined deformation-band has an oriented structure inclined to the tensile axis. By use of 

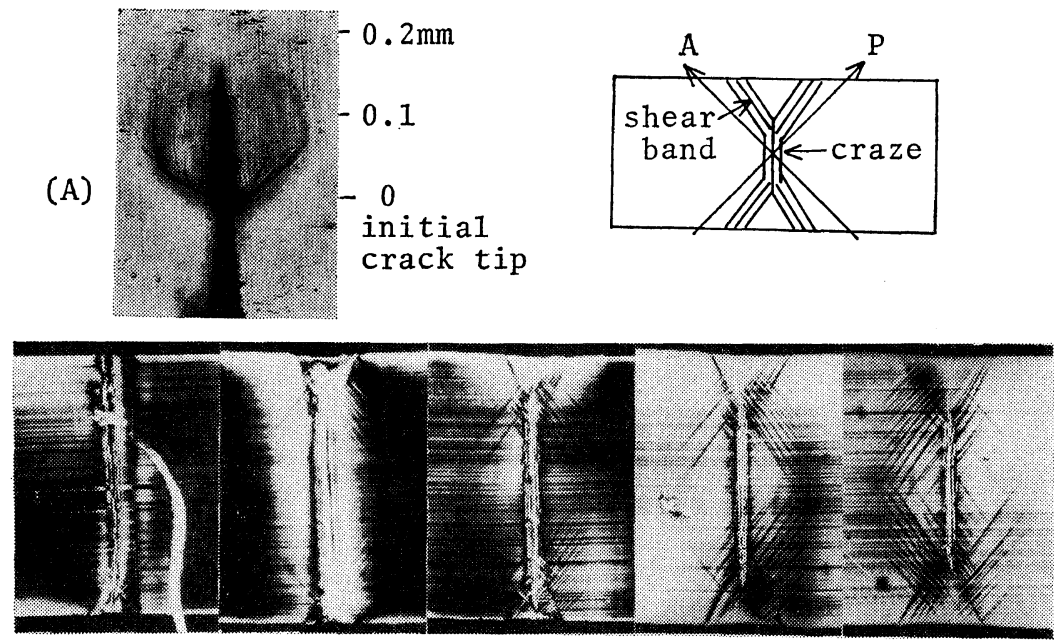

$0.01(\mathrm{~mm})$

0.03

0.04

0.06

0.08

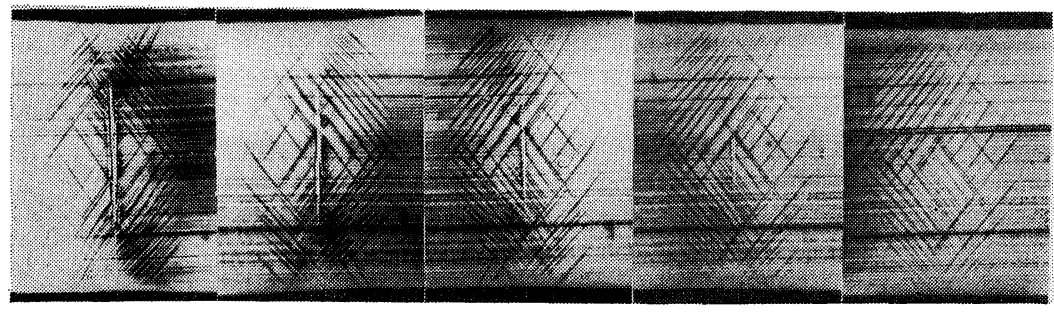
0.10
0.12
0.14
0.16
0.18

Figure 4. Microphotographs of the overall view of the plastic-zone (A) and its successive cross-sections $(\mathrm{Cn})$ from the initial crack tip in the first step. The cross-sections were observed between cross polars which were inclined $45^{\circ}$ to the tensile axis: A, analyser; $\mathbf{P}$, polarizer.

an interference microscope, it was found that the density of the material within the perpendicular deformation-band was smaller than that of the undeformed region, but the density within the inclined deformation-bands was either equal or slightly greater than that of the surrounding material. These facts indicate that the perpendicular deformation-band is a craze structure which contains many voids and that each inclined deformation-band is a shear band. This observation is closely similar to that reported for unaxially compressed samples. ${ }^{6-8}$

Figure 5 shows a new mode of deformation in the second step. The specimen thickness ahead of the crack tip is strongly reduced and this apparent thinning is found at a distance as great as $1.12-1.14 \mathrm{~mm}$. In this region, as may be observed, the shear bands which are inclined in the opposite directions intersect each other at the center of the specimen although individual shear band is indistinguishable at the immediate vicinity of the crack tip. These intersections occupy the whole thickness of the specimen and this region corresponds to that which is observed as a homogenous zone. Beyond this region, the reduction in the specimen thickness gradually decreases corresponding to a decrease in the number of the intersections. The extinction direction in the region of the intersection coincides with that of the tensile axis.

\section{Birefringence of the Plastic-Zone}

Figure 6 shows the inclination angle of extinction direction of the interior of the shear bands obtained from the observation on the cross-sections. Here, the inclination angle was chosen so that it corresponded to the direction 
Analysis of the Localized Plastic Deformation

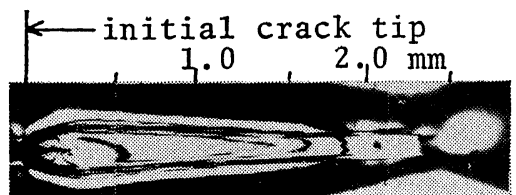

(A)

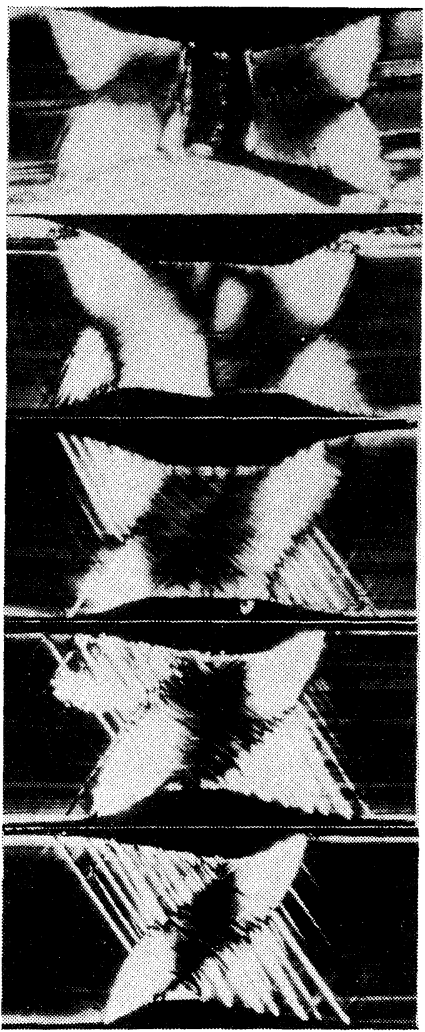

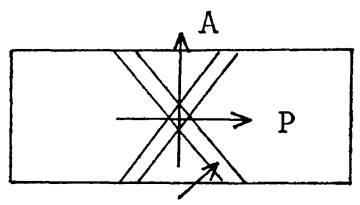

shear band

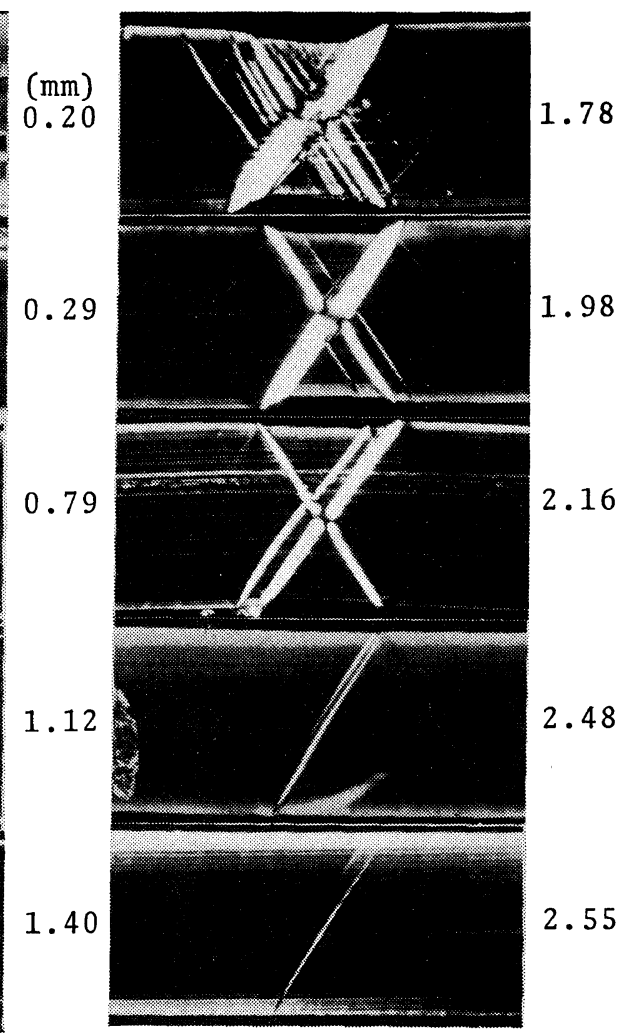

Successive cross-sections

Figure 5. Microphotographs of the overall view of the plastic-zone (A) and its successive cross-sections $(\mathrm{Cn})$ from the initial crack tip in the second step. The cross-sections were observed between cross polars where the polarizer was parallel to the tensile direction: A, analyser; $\mathbf{P}$, polarizer.

of maximum refractive index. As may be seen, the inclination angle of the extinction direction slightly decreases on approaching the crack tip. Figure 7 shows the birefringence of the interior of the shear band and of the intersection region for the same specimen shown in Figure 6. Birefringence in the shear bands is not great although the width of the shear bands increases as the distance from the initial crack tip decreases. In the region of intersection of the shear bands the birefringence is higher than that of the shear band. This indicates that the intersection causes the changes in orientation direction and birefringence at the same time.

Figure $8(b)$ shows a cross-section parallel to the direction of propagation of the plastic-zone (Figure 8(a)). In region A variation of isochromatic fringes can scarcely be observed except in the immediate vicinity of the crack tip (Figure $8(\mathrm{c}))$, and it corresponds to the plastic-zone where intersections of the shear bands occur everywhere. A variation of the isochromatic fringe contour and a reduction in thickness of the specimen are observed in region $B$. In region 


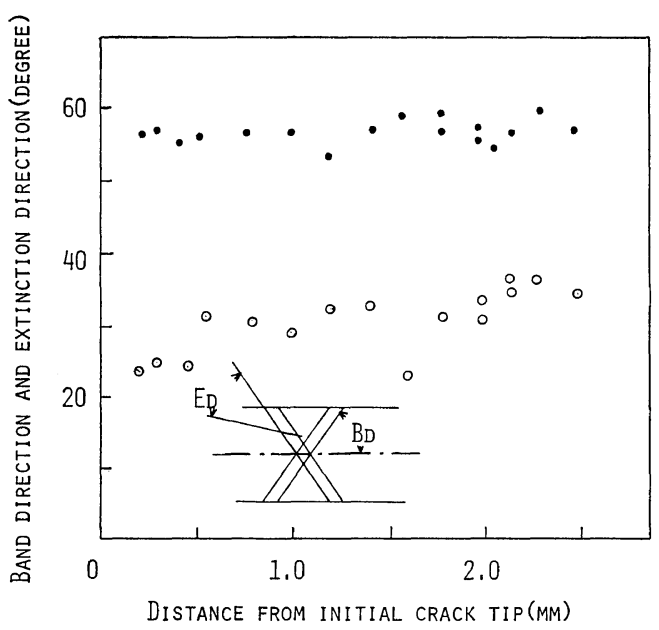

Figure 6. Angle of extinction direction of the shear bands: $\mathrm{ED}(\bigcirc)$, extinction direction with respect to the direction of the shear bands; $\mathrm{BD}(\mathrm{O})$, direction of the shear bands with respect to the tensile axis.

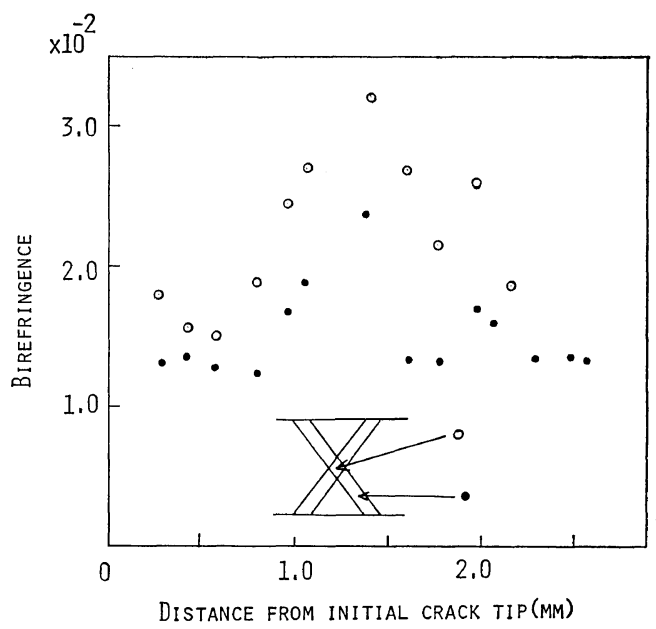

Figure 7. Birefringence of the shear bands and the intersecting regions of the shear bands for the same specimen in Figure 6: (O), shear band; $(O)$, region of intersection.

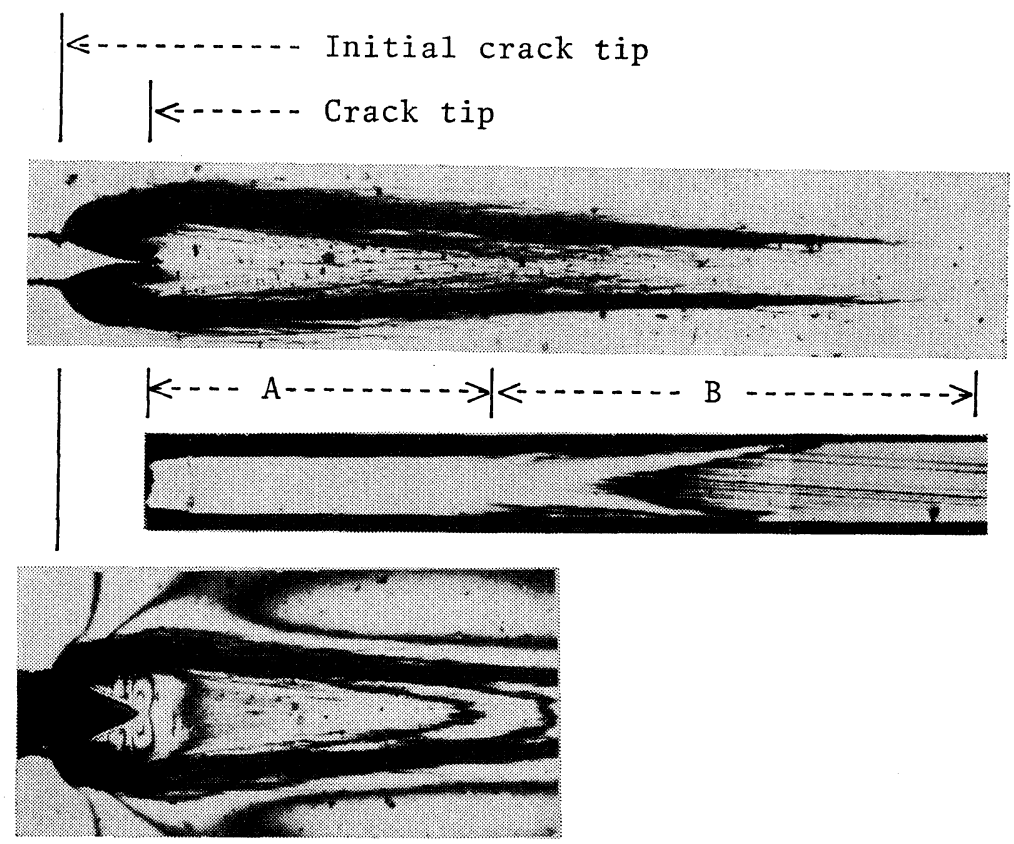

Figure 8. Microphotographs of the cross-section (Cp): The cross-section was observed between cross polars inclined $45^{\circ}$ to the progressive direction of the plastic-zone: (a), normal microscopic observation of the plastic-zone; (b), cross-section of (a), (c), photoelastic observation of (a).

A the extinction direction corresponds to the progressive direction of the plastic-zone and the birefringence shows a uniform value of $8.9 \times$
$10^{-3}$. In the bright region in $\mathbf{B}$ the extinction direction inclines $10^{\circ}$ to the progressive direction and the birefringence is $7.3 \times 10^{-3}$. 
Analysis of the Localized Plastic Deformation

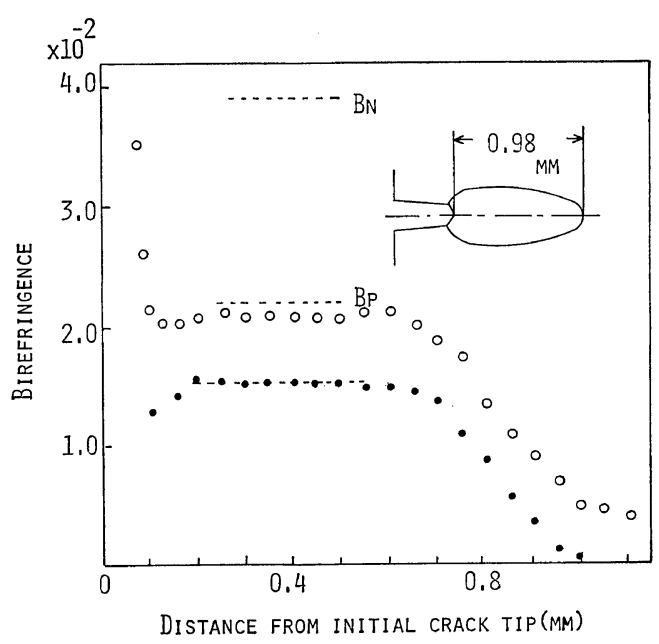

Figure 9. Birefringence of the plastic-zone along the initial crack: ( $\bigcirc$ ), loading. (O), unloading. Bp, birefringence calculated from the true thickness; $\mathrm{Bn}$, birefringence obtained with necking.

The apparent birefringences of the plastic-zone when loaded and unloaded are shown in Figure 9 as a function of distance from the crack tip. They have been calculated from the value of retardation divided by the original thickness of the specimen. The value in the presence of load is higher than that in the absence of load, but both of them show the same type of variation except near the crack tip. The thickness of the specimen in the region of constant birefringence has been uniformly reduced to approximately $70 \%$ of the original thickness. The birefringence calculated from this thickness is still smaller than that after necking as shown in Figure 9.

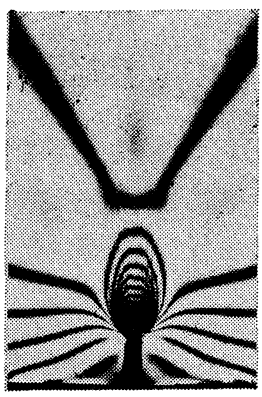

(A)

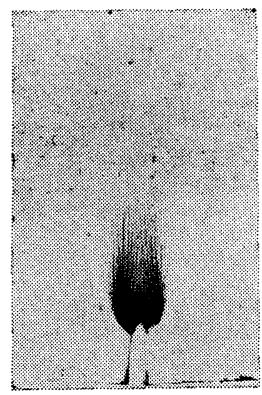

(B)
Figure 10. Isochromatic fringes (A) and plasticzone (B) for the photoelastic analysis.

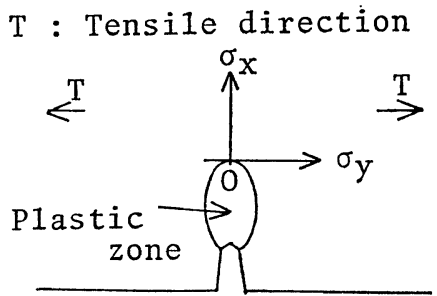

Figure 11. Definition of the stress components.

Estimation of Stress Distribution Ahead of the Plastic-Zone

The stress distribution ahead of the plasticzone tip is estimated photoelastically. Figure 10 shows the shape of the plastic-zone and the isochromatic fringes. Figure 12 shows the stress components $\sigma_{x}$ and $\sigma_{y}$ which are defined in Figure 11. It is found that both $\sigma_{x}$ and $\sigma_{y}$ increase on coming close to the plastic-zone tip. In the immediate vicinity of the tip of the plastic-zone, $\sigma_{y}$ and $\sigma_{x}$ are $8.5 \mathrm{~kg} / \mathrm{mm}^{2}$ and 3.4 $\mathrm{kg} / \mathrm{mm}^{2}$, respectively. The yield under uniaxial tensile test occurs at a stress of $6.0 \mathrm{~kg} / \mathrm{mm}^{2}$. It should be noted that $\sigma_{y}$ ahead of the plasticzone is considerably higher than the tensile yield stress.

\section{DISCUSSION AND CONCLUSIONS}

The observations of the cross-sections and shapes of the plastic-zone show that the plasticzone consists of crazes, shear bands and their

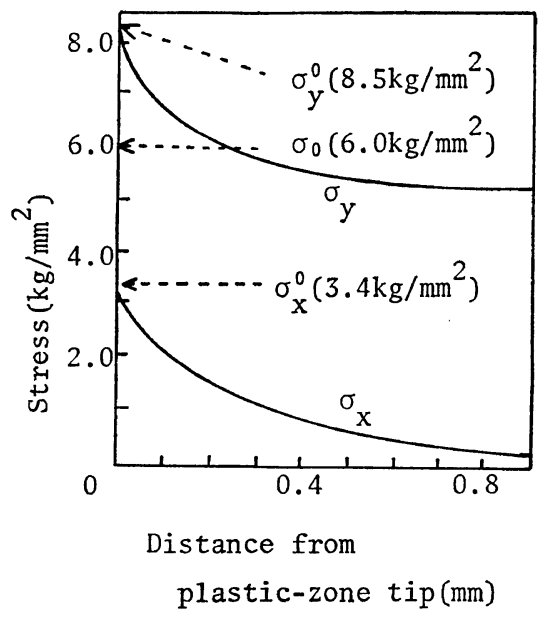

Figure 12. $\sigma_{x}$ and $\sigma_{y}$ along the initial crack: $\sigma_{0}$, tensile yield stress; $\sigma_{x}{ }^{0}$ and $\sigma_{y}{ }^{0}$, stresses ahead of plastic-zone. 

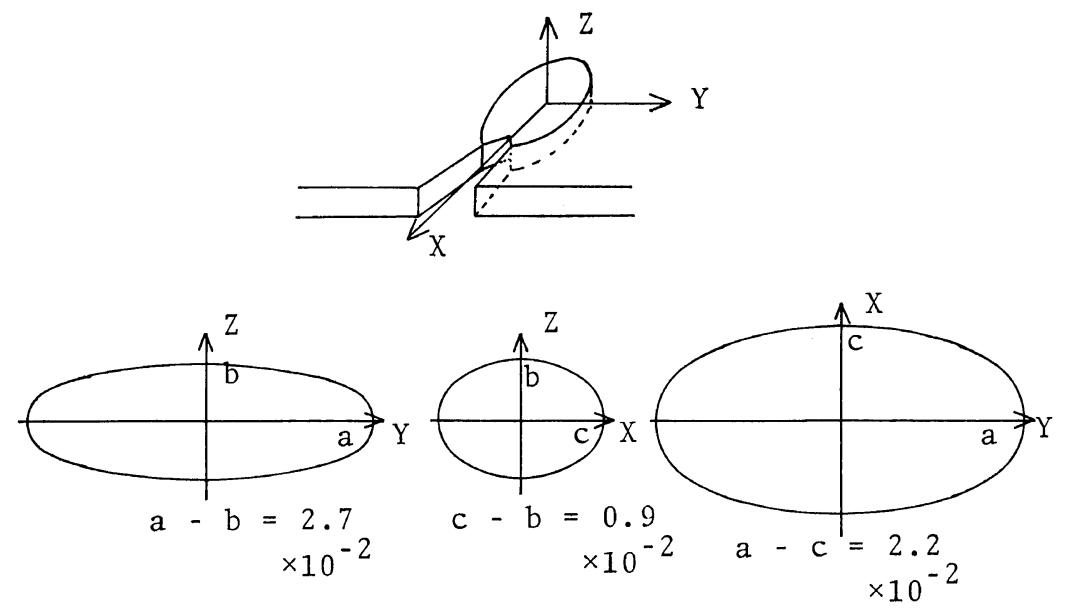

Figure 13. Birefringences in each direction, which were summarized from Figure 7, Figure 9 and the birefringence in the cross-section (b) shown in Figure 8.

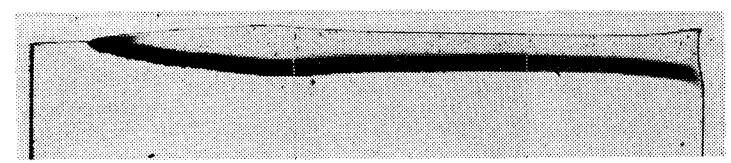

Figure 14. Plastic-zone in the fracture specimen.

intersections. When the stress is applied, the crazes are initially formed at the crack tip. Following development of the crazes, the shear bands initiate from the surface near the crack tip and extend to a region some distance from the initial crack tip.

As shown in Figure 12, $\sigma_{y}$ ahead of the plasticzone is considerably higher than the tensile yield stress. This fact indicates that the stress component $\sigma_{y}$ is affected by the other stress component $\sigma_{x}$ which is due to the restrain of strain in $x$-direction. Therefore, as speculated from the birefringence shown in Figure 13, these biaxial stresses cause biaxial orientation of the molecular chain in the structure of the shear bands and their intersections. Consequently, the structure of the plastic-zone of the crack tip is essentially different from that which would be produced through a unaxial tensile deformation (see Figure 9).

Since the change in density of the material within the shear bands is negligible, and the

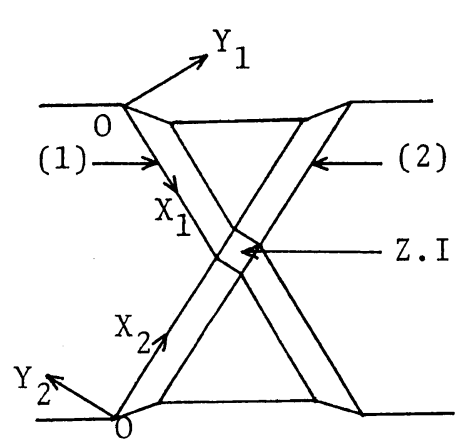

(a)

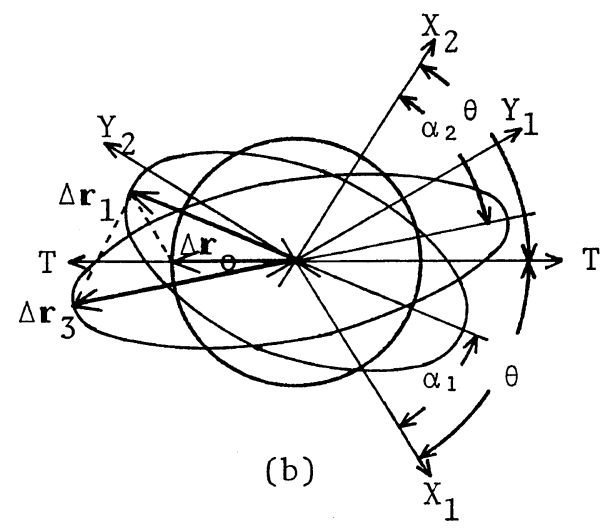

(b)

\section{Z.I : Zone of intersection \\ $\mathrm{T}$ : Tensile direction}

Figure 15. (a), definition of coordinate system; (b), variation of strain ellipsoid by simple shear strain. 
strain in the progressive direction of the plasticzone is approximately zero as shown in Figure 14 , it is concluded that the initiation of shear bands is caused by simple shear. The plastic strain in the shear band can be determined from the extinction direction on the basis of the concept of strain ellipsoid which has been suggested by Ward. ${ }^{9}$ We consider the initial shear band (1) which is shown in Figure 15(a). The vector $\Delta r_{0}$ which connects two points close to one another in the base circle in an undeformed state can be related to the vector $\Delta r_{1}$ in a deformed state by the displacement tensor $\gamma_{1}$. A coordinate system $\left(O X_{1}, O Y_{1}, O Z_{1}\right)$ is introduced such that $O X_{1}$ is parallel, $O Y_{1}$ is perpendicular to the shear direction and $O Z_{1}$ is in the film plane. If the magnitude of the simple shear is $\gamma$, the displacement tensor $\gamma_{1}$ is given by

$$
\gamma_{1}=\left[\begin{array}{lll}
1 & \gamma & 0 \\
0 & 1 & 0 \\
0 & 0 & 1
\end{array}\right]
$$

The strain tensor $(\lambda)$ is defined by $\lambda=\gamma_{1} \cdot \gamma_{1}{ }^{+}$. Therefore, for this deformation the strain ellipsoid is given by

$$
\Delta r_{1}{ }^{+} \cdot \lambda^{-1} \cdot \Delta r_{1}=1
$$

The principal extension ratios $\left(\sqrt{\lambda_{1,2}}\right)$ of the strain ellipsoid after shearing in shear band (1) are

$$
\lambda_{1,2}=1+\frac{1}{2} \gamma\left(\gamma \pm \sqrt{\gamma^{2}+4}\right)
$$

and the angle $\alpha_{1}$ between the direction of the shear band and principal axis of the sheared ellipsoid is given by

$$
\cot \left(2 \alpha_{1}\right)=\frac{\gamma}{2}
$$

Since the angle of extinction direction which is shown in Figure 6 decreases on coming close to the crack tip, the shear strain calculated from eq 4 increases. But the birefringence of the shear bands does not exhibit such a tendency. Therefore, the variation of extinction direction seems to be caused by the compression stress which is applied to the plastic-zone by the restoration of the elastically deformed region when the stress is removed. At the tip of the plasticzone the angle of the extinction direction is found to be $35^{\circ}$ which corresponds to a shear strain of about 0.73 and to a maximum princi- pal extension strain $\left(\sqrt{\lambda_{1}}-1\right)$ of 0.43 . Using the tubular sample in shear test, Turner ${ }^{10}$ has shown that the strain within the shear band is a uniform shear strain of 0.7 .

When a new shear band (2) which is inclined in the opposite direction to the initial shear band (1) is formed, we assume that the new band has shear strain of opposite sign but equal magnitude to the initial shear band. In order to see the result of the intersection of these bands, a new coordinate system $\left(\mathrm{OX}_{2}, \mathrm{OY}_{2}, \mathrm{OZ}_{2}\right)$ is introduced as shown in Figure 15a. On this new coordinate system the strain ellipsoid for the initial shear band is given by

$$
\Delta r_{2}{ }^{+} \cdot\left(T \cdot \lambda^{-1} \cdot T^{+}\right) \cdot \Delta r_{2}=1
$$

where $\Delta r_{2}$ is the vector in the new coordinate system and $T$, matrix of transformation, is given by

$$
T=\left[\begin{array}{ccc}
\cos 2 \theta & \sin 2 \theta & 0 \\
-\sin 2 \theta & \cos 2 \theta & 0 \\
0 & 0 & 0
\end{array}\right]
$$

where $\theta$ is the angle of the shear band to the tensile direction. From the above assumption, the displacement tensor $\left(\gamma_{2}\right)$ in the new coordinate system is given by

$$
\gamma_{2}=\left[\begin{array}{ccc}
1 & -r & 0 \\
0 & 1 & 0 \\
0 & 0 & 1
\end{array}\right]
$$

The final strain ellipsoid is given by

$$
\Delta r_{3}{ }^{+} \cdot\left(\gamma_{2} \cdot T \cdot \lambda \cdot T^{+} \cdot \gamma_{2}{ }^{+}\right)^{-1} \cdot \Delta r_{3}=1
$$

where $\Delta r_{3}$ is the vector after the new deformation occurs. The principal extension ratios $\left(\sqrt{\lambda_{1,2}^{*}}\right)$ of the final strain ellipsoid are

$$
\begin{aligned}
\lambda_{1,2}^{*}= & \frac{2+\sin ^{2} 2 \theta \gamma^{2}\left(4+\gamma^{2}\right)}{2} \pm \frac{\sin 2 \theta \gamma}{2} \\
& \times\left\{\sqrt{\sin ^{2} 2 \theta \gamma^{4}\left(\gamma^{2}+8\right)+4 \gamma^{2}\left(4 \sin ^{2} 2 \theta+1\right)+16}\right\}
\end{aligned}
$$

and the angle $\alpha_{2}$ between the shear direction $\left(O X_{2}\right)$ and the principal axis of the sheared ellipsoid is given by

$$
\cot \left(2 \alpha_{2}\right)=\frac{1}{2}\left(\frac{4 \cot 2 \theta+2 \gamma+\gamma^{3}}{-2+\gamma \cot 2 \theta-\gamma^{2}}\right)
$$

Since the angle $\theta$ is about $58^{\circ}$, the angle $\alpha_{2}$ 
is $-45^{\circ}$ which corresponds to an angle of $13^{\circ}$ to the tensile axis in an anticlockwise direction (see Figure 15b), and the maximum principal extension strain is 0.92 . When the calculation of the extinction direction is compared with the experimental results, it is found that the calculated direction does not agree with the measured direction which corresponds to the tensile direction. The maximum strain in the plastic-zone can be estimated from the decrease in thickness under the assumption of constant volume, and this is approximately 0.43 . The calculated value from eq 9 is significantly higher than this value. The plastic strain calculated from the thickness of the plastic-zone corresponds well with the maximum principal strain at the initial shear band rather than that calculated from eq 9 . These results indicate that the increase in the plastic strain within the first shear band is forced to stop due to the orientation hardening. Therefore, it appears that the plastic deformation relating to the intersection of shear bands corresponds to reorientation of the molecular chains in the first shear band without further extension of them. It appears that future research on this subject should be aimed at examining the shear deformation of the oriented polymer.

Acknowledgment. The authors wish to thank Mr. T. Murayama for his assistance in the experimental work. The authors are also indebted to Teijin Co. for supplying polycarbonate films.

\section{REFERENCES}

1. I. Narisawa, M. Ishikawa, and H. Ogawa, Polymer J., 8, 181 (1976).

2. S. S. Sternstein and L. Ongchin, J. Amer. Chem. Soc. Polym. Prep., 10, 1117 (1969).

3. A. S. Argon, Phil. Mag., 28, 839-864 (1973).

4. P. I. Vincent "Deformation and Fracture of High Polymer," Plenum Press, New York N.Y., 1973, p 287.

5. H. F. Brinson, Exp. Mech., Feb. 72 (1970).

6. P. B. Bowden and S. Raha, Phil. Mag., 22, 473 (1970).

7. A. S. Argon, J. Appl. Phys., 39, 1899 (1968).

8. L. Camwell and D. Hull, Phil. Mag., 27, 1135 (1973).

9. N. Brown, R. A. Ducker, and I. M. Ward, Brit. J. Appl. Phys., 1, 1369 (1968).

10. W. Wu and A. P. Tuner, J. Polym. Sci., 11, 2199 (1973). 